\title{
Educación ambiental y la evaluación de residuos plásticos durante la pandemia en distrito de Barranca
}

\author{
Environmental education and the evaluation of plastic waste during the pandemic in the district of Barranca
}

Educação ambiental e avaliação de resíduos plásticos durante pandemia no distrito de Barranca

\section{Magna Guzmán Avalos ${ }^{1}$ \\ mguzmana@unasam.edu.pe \\ https://orcid.org/0000-0002-4038-3507}

Dante Elmer, Sánchez Rodríguez
dsanchezr@unasam.edu.pe
https://orcid.org/0000-0002-6810-6253
José Yovera Saldarriaga ${ }^{1}$

jyoveras@unasam.edu.pe

https://orcid.org/0000-0001-5235-0270

Jesús Manuel More López ${ }^{1}$

mamore_08_31@hotmail.com

https://orcid.org/0000-0003-0315-9810

\section{Dante Daniel Cruz Nieto ${ }^{2}$}

daniel2262@hotmail.com

https://orcid.org/0000-0003-0052-5619

\author{
${ }^{1}$ Universidad Nacional Santiago Antúnez de Mayolo, Huaraz-Perú \\ ${ }^{2}$ Universidad Nacional José Faustino Sánchez Carrión, Huaura-Perú
}

Artículo recibido 25 de octubre 2021, arbitrado y aceptado 15 de noviembre 2021 y publicado 06 de enero 2022

\section{RESUMEN}

La investigación tuvo como objetivo determinar la cantidad de residuos de plásticos generados durante la pandemia. Se basa en la metodología descriptiva longitudinal, en donde se empleó el método de Kunitoshi Sakurai y se elaboraron cuestionarios sobre qué actitud toman al momento de reciclar, caracterización y cuantificación de residuos. Los resultados determinaron que en noviembre sobresalió con $57.32 \%$, per cápita de residuos sólidos domiciliarios el jueves con 0.49 $\mathrm{kg} /$ habitante/ día, porcentaje de residuos plásticos por mes con $7.48 \%$ en noviembre, porcentaje en relación al per cápita el viernes con $10.85 \%$ y actitud de reciclar con $61 \%$ no reciclan. Se concluye que, en noviembre destacó con $7.48 \%$; es decir que en $100 \mathrm{Kg}$ de residuos $7.48 \mathrm{Kg}$ son plásticos. Por lo tanto, se establece como un indicador porcentual, para que se tome conciencia ecológica al momento de reciclar con el fin de reducir la contaminación.

Palabras clave: Residuos plásticos; Caracterización; Actitud ecológica; Reciclar; Contaminación ambiental

\section{ABSTRACT}

The research aimed to determine the amount of plastic waste generated during the pandemic. It is based on the longitudinal descriptive methodology, where the Kunitoshi Sakurai method was used and questionnaires were elaborated on what attitude they take when recycling, characterization and quantification of waste. The results determined that in November it stood out with 57.32\%, per capita of household solid waste on Thursday with $0.49 \mathrm{~kg} /$ inhabitant / day, percentage of plastic waste per month with $7.48 \%$ in November, percentage in relation to per capita on Friday with $10.85 \%$ and attitude to recycle with $61 \%$ do not recycle. It is concluded that, in November it stood out with 7.48\%; In other words, in $100 \mathrm{Kg}$ of waste, $7.48 \mathrm{Kg}$ are plastics. Therefore, it is established as a percentage indicator, so that ecological awareness is taken when recycling in order to reduce pollution.

Key words: Plastic waste, Characterization; Ecological attitude; Recycle; Environmental pollution

\section{RESUMO}

A pesquisa teve como objetivo determinar a quantidade de resíduos plásticos gerados durante a pandemia. Baseia-se na metodologia descritiva longitudinal, onde foi utilizado o método Kunitoshi Sakurai e elaborados questionários sobre as atitudes que tomam na reciclagem, caracterização e quantificação dos resíduos. Os resultados determinaram que em novembro se destacou com 57,32\%, per capita de resíduos sólidos domiciliares na quinta-feira com 0,49 kg / habitante / dia, percentual de resíduos plásticos por mês com 7,48\% em novembro, percentual em relação ao per capita na sexta-feira com $10,85 \%$ e atitude para reciclar com $61 \%$ não reciclam. Conclui-se que, em novembro destacou-se com 7,48\%; Ou seja, em $100 \mathrm{Kg}$ de resíduo, 7,48 Kg são plásticos. Portanto, é estabelecido como um indicador percentual, para que haja consciência ecológica na hora de reciclar para reduzir a poluição.

Palavras-chave: Resíduos de plástico; Caracterização; Atitude ecológica; Reciclagem; Poluição ambiental 


\section{INTRODUCCIÓN}

En este segundo año de la pandemia de la Covid-19, se percibió el incremento de materiales de plásticos como bolsas, envases, empaques y que se utilizaron más seguidos en centros comerciales, mercados y otros lugares de abastos. Según el Programa de las Naciones Unidas para el Medio Ambiente (2019), expone que el plástico es un material ligero, higiénico y resistente que se puede moldear de distintas maneras y utilizar en una amplia gama de aplicaciones.

Asimismo, se menciona que la preferencia de estos materiales de plásticos ha tenido gran importancia en la pandemia; pues su uso ha favorecido en el traslado, conservación e higiene de los productos; sin embargo, el continuo uso de estos materiales ha ocasionado una problemática social sobre el manejo de estos residuos. Este análisis se sostiene con Flores (2020) quien menciona que el impacto de la pandemia de la Covid-19 ha generado el efecto contrario, un incremento en el uso generalizado de productos descartables plásticos, como envases de alimentos y dispositivos médicos como mascarillas, guantes, trajes de protección, entre otros, tanto de origen hospitalario como doméstico.

Debido a esta situación es necesario tener en cuenta la adecuada disposición final de los residuos de plásticos, para lo cual debe depositarlos en tachos, reciclarlo, usar materiales de plástico en lo necesario y sobre todo tener conciencia ambiental. Estas actitudes conllevarán a reducir la contaminación ambiental y la propagación de enfermedades; puesto que estos residuos demoran muchos años en degradarse Jaén et al. (2019) exponen que el plástico es un material que ha pasado a ser parte fundamental de vida, pero quizá sus repercusiones no son bien comprendidas por la sociedad. Desde el ámbito educativo, los problemas generados por su consumo, no deben dejar de ser indiferentes, entre otras razones, porque los educadores deben proporcionar y promocionar tareas de concientización ciudadana que permitan afrontar las posibles amenazas para la vida en el planeta.

También, es necesario mencionar que los materiales plásticos están hechos por componentes químicos derivados del petróleo; lo cual se usa como bolsas, empaques, envases etc. Por lo que, se debe tener conciencia ambiental; es decir reciclar, usar lo necesario u otras bolsas hechas de materiales degradables; puesto que estos materiales demoran muchos años en degradarse y que han ocasionado contaminación al ecosistema marino y directamente ha afectado a la población, por el consumo de productos marinos, pues en muchas investigaciones se concluye que se han encontrado micropartículas de plásticos que son nocivos a la salud. Según, García, y Rentería, (2019), manifiestan que los plásticos son materiales maleables generalmente derivados del petróleo, aunque también los existen derivados de otras fuentes como de productos vegetales. Existen variedades de productos para distintas necesidades, sobre todo para envases y empaques. Asimismo, López-Aguirre et al., (2020) mencionan que los plásticos tardan alrededor de 180 años en degradarse y son utilizados en las industrias, en la vida cotidiana son productos con una limitada capacidad de autodestrucción, en consecuencia, quedan durante muchos años como residuos. 
Por este motivo, se realizó la investigación sobre la educación ambiental y la evaluación de residuos de plásticos durante la pandemia en el distrito de Barranca, lo cual se tuvo como objetivo determinar la cantidad de residuos de materiales de plásticos y que actitudes toman la población al momento de reciclar. Obtenidos los datos se procesaron con estadísticas básicas y los resultados permitieron conocer el diagnóstico de estos residuos y tomar medidas para reducir los daños ambientales ocasionados por estos residuos.

\section{MÉTODO}

Se realizó una investigación descriptiva de corte longitudinal que estudia una o más variables a lo largo de un periodo, que varía según el problema investigado y las características de la variable que se estudia (Pineda et al, 1994). Por lo que, se evaluó sobre las actitudes y características de los residuos, para lo cual se empleó cuestionarios. La población en estudio, estuvo constituido por los habitantes del distrito de Barranca que habitan en zonas rurales y urbanas conformados por 71383 habitantes (INEI, 2016). Asimismo, estos habitantes se establecen en 17129 viviendas según el último censo (INEI, 2017). Concerniente a la muestra, se obtuvo 50 casas, de cada casa se evaluó caracterizó y cuantificó los residuos. También se hicieron preguntas a una persona por casa el último mes. Cabe mencionar que se obtuvo la muestra aplicando la fórmula de Kunitoshi Sakurai, que está publicado por el Centro Panamericano de Ingeniería Sanitaria y Ciencias del Ambiente (CEPIS), tal como se muestra en la siguiente expresión (Cantanhede, et. al. 2005).

\section{Fórmula:}

$$
n=\frac{\left(Z_{1-\frac{\alpha}{2}}^{2}\right)(N)\left(\sigma^{2}\right)}{(N-1) E^{2}+\left(Z_{1-\frac{\alpha}{2}}^{2}\right)\left(\sigma^{2}\right)}
$$

Dónde:

$\mathrm{N}=$ Número de viviendas $=17129$ casa (INEI, 2017).

$$
Z_{1-\frac{\alpha}{2}}^{2}=1.96
$$

(El coeficiente de confianza $\left(1-\frac{\propto}{2}\right)$ del $95 \%$ en valores de generación de residuos)

$\mathrm{E}=0.056 \mathrm{~kg} / \mathrm{hab}$., por día. (Del error se permite el $10 \%$ de $0.56 \mathrm{Kg} / \mathrm{hab}$, por día). Por lo tanto, la estimación nacional es $0.56 \mathrm{~kg} / \mathrm{hab}$., por día.

$\sigma=0.2$, la desviación estándar (La variación de generación de residuos de casa)

$\mathrm{n}:$ muestra $=50$ casas

Se obtuvo la recolección de datos de los residuos domiciliaros de cada casa, aplicando instrumentos de observación y anotación de datos en cuadernillos. Este procedimiento consto de evaluar, caracterizar y cuantificar los componentes de los residuos por día, semana y mes.

En cuanto a la recolección de datos de las personas se entrevistó a una sola persona por casa en un mes, para lo cual se empleó instrumentos de cuestionarios. Se determinó la muestra empleando la metodología de Kunitoshi Sakurai; para lo cual 
se tomó en cuenta la cantidad de viviendas y se procesó con la fórmula de muestra finita se obtuvo 50 casas. Luego se hizo la evaluación de manera aleatoria a la fuente; es decir, se separó los residuos sólidos orgánicos, inorgánicos e inertes de las bolsas o tachos y se cuantificó obteniéndose el per cápita, por semana, por mes y el porcentaje de residuos plásticos por per cápita. También se preguntaron y evaluaron los datos del mes de noviembre como mes representativo sobre ¿Qué actitud toma al momento de reciclar los residuos de plásticos? Por último, recabados los datos se procesaron mediante estadísticas básicas y los resultados obtenidos se interpretaron y analizaron.

\section{RESULTADOS}

\section{Caracterización de los residuos domiciliarios}

De acuerdo a la evaluación de las características de los residuos domiciliarios que se detalla en la Tabla 1, se determinó que hubo incremento en la generación de los residuos domiciliarios en el mes de noviembre con $1591.77 \mathrm{~kg} / 50$ casas/ mes. Asimismo, se nota el aumento de residuos orgánicos con $57.32 \%$ con relación a los demás meses. Por lo que, significa que hubo incremento de la generación de residuos domiciliarios y residuos orgánicos debido a la demanda del consumo en mercados y abastecimiento de productos de primera necesidad.

Tabla 1. Caracterización de los residuos domiciliarios Kg/ mes.

\begin{tabular}{|c|c|c|c|c|c|c|c|}
\hline \multirow{2}{*}{ Tipo de residuos } & \multirow{2}{*}{ Tipo de residuos } & \multicolumn{2}{|c|}{ Setiembre } & \multicolumn{2}{|c|}{ Octubre } & \multicolumn{2}{|c|}{ Noviembre } \\
\hline & & $\mathrm{Kg}$ & $\%$ & Kg & $\%$ & $\mathrm{Kg}$ & $\%$ \\
\hline \multirow[t]{10}{*}{ Residuos No Aprovechables } & Residuos inertes & 54.23 & 3.46 & 50.25 & 3.31 & 55.25 & 3.47 \\
\hline & Tierra & 54.23 & 3.46 & 50.25 & 3.31 & 55.25 & 3.47 \\
\hline & Residuos inorgánicos & 626.60 & 39.94 & 606.71 & 39.99 & 624.17 & 39.21 \\
\hline & Mascarillas & 22.58 & 1.44 & 23.58 & 1.55 & 20.55 & 1.29 \\
\hline & Viseras & 17.51 & 1.12 & 13.51 & 0.89 & 9.85 & 0.62 \\
\hline & Jeringas & 10.50 & 0.67 & 8.58 & 0.57 & 7.25 & 0.46 \\
\hline & Blísteres de pastillas & 12.58 & 0.80 & 10.85 & 0.72 & 9.25 & 0.58 \\
\hline & Ampollas de vidrio & 16.39 & 1.04 & 13.39 & 0.88 & 10.23 & 0.64 \\
\hline & Pilas y baterías & 49.85 & 3.18 & 44.26 & 2.92 & 41.23 & 2.59 \\
\hline & Residuo sanitario & 66.23 & 4.22 & 60.36 & 3.98 & 70.23 & 4.41 \\
\hline \multirow[t]{10}{*}{ Residuos Aprovechables } & Papeles y cartones & 218.13 & 13.90 & 208.42 & 13.74 & 215.52 & 13.54 \\
\hline & Plásticos & 107.90 & 6.88 & 112.93 & 7.44 & 116.82 & 7.34 \\
\hline & Metales & 36.85 & 2.35 & 38.36 & 2.53 & 44.36 & 2.79 \\
\hline & Vidrios (botellas) & 43.61 & 2.78 & 45.62 & 3.01 & 49.25 & 3.09 \\
\hline & Envases Tetra pack & 24.47 & 1.56 & 26.85 & 1.77 & 29.63 & 1.86 \\
\hline & Residuos orgánicos & 888.12 & 56.61 & 860.29 & 56.70 & 912.35 & 57.32 \\
\hline & Residuos de cocina & 638.30 & 40.68 & 620.30 & 40.88 & 646.36 & 40.61 \\
\hline & Residuos de frutas & 97.86 & 6.24 & 92.36 & 6.09 & 111.36 & 7.00 \\
\hline & Residuos de jardines & 151.96 & 9.69 & 147.63 & 9.73 & 154.63 & 9.71 \\
\hline & Total & 1568.95 & 100.0 & 1517.25 & 100.0 & 1591.77 & 100.0 \\
\hline
\end{tabular}


Per cápita de residuos sólidos domiciliarios

Respecto a la evaluación per cápita de los residuos domiciliarios que se detalla en la Figura 1, se observa que el día jueves con $0.49 \mathrm{~kg} /$ habitante $\mathrm{kg} /$ habitante sobresalió con relación a los demás días. Este resultado, se analizó que al finalizar la semana se notó un incremento de frecuencia a los mercados, restaurantes y otros lugares de comercio; lo cual resultó el aumento de residuos.

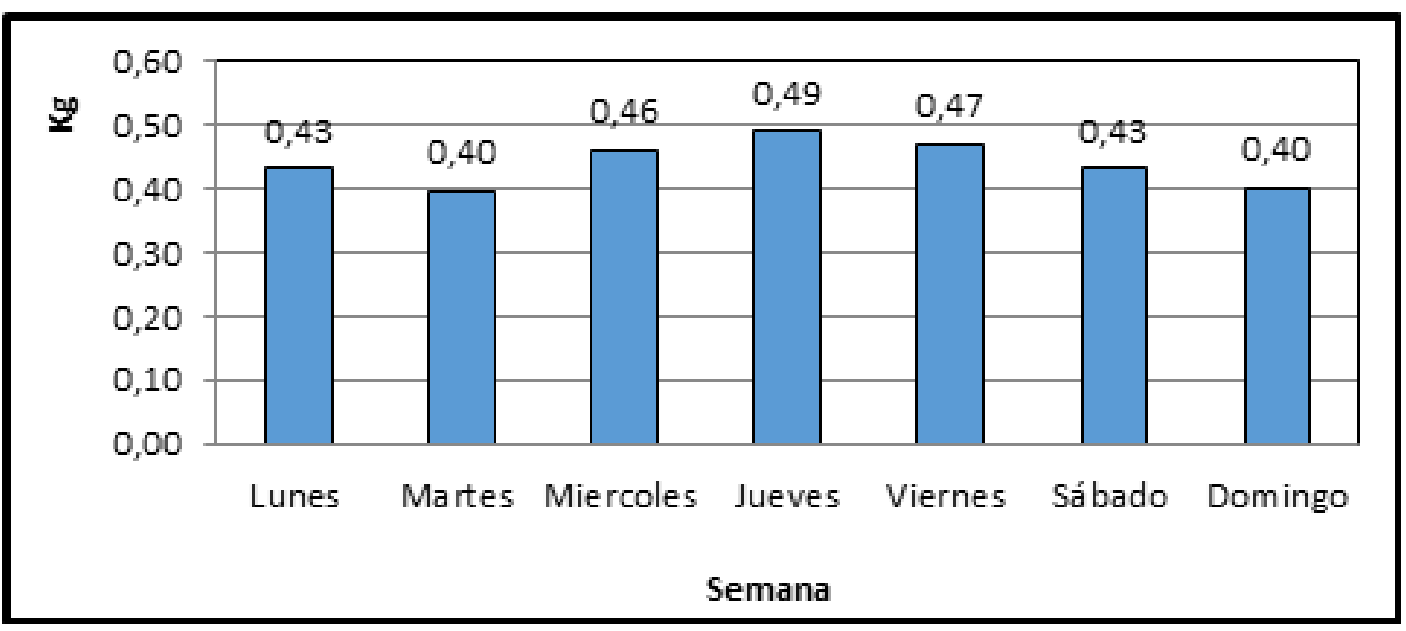

Figura 1. Per cápita de residuos sólidos domiciliarios.

\section{Porcentaje de residuos de plásticos por mes}

En cuanto al porcentaje de residuos de plásticos por mes que se indica en la Tabla 2, se observa que en noviembre destacó con $7.48 \%$ con relación a los demás meses. Este resultado se interpreta que al finalizar el año se percibió la concurrencia a centros comerciales, mercados, restaurantes y otros lugares de comercio, lo cual incrementó los residuos de materiales de plásticos, restos de comida y otros.

Tabla 2. Porcentaje de residuos de plásticos por mes.

\begin{tabular}{cccc}
\hline Cantidad & Setiembre & Octubre & Noviembre \\
\hline Peso $(\mathrm{kg})$ & 107.90 & 112.93 & 116.82 \\
Peso total $(\mathrm{kg})$ & 1568.95 & 1517.25 & 1560.77 \\
Porcentaje & 6.88 & 7.44 & 7.48 \\
\hline
\end{tabular}

\section{Porcentaje de residuos de plásticos en relación} al per cápita

En el porcentaje de residuos de plásticos en relación al per cápita que se observa en la Figura 2, se aprecia que el viernes destacó con $10.85 \%$ de residuos de plásticos en relación al per cápita.
Este resultado es el indicador que evidencia que al finalizar la semana se percibe la continua frecuencia a lugares comerciales; sin embargo, es necesario tener en cuenta la adecuada disposición final de los residuos con la finalidad de no contaminar el ambiente. 


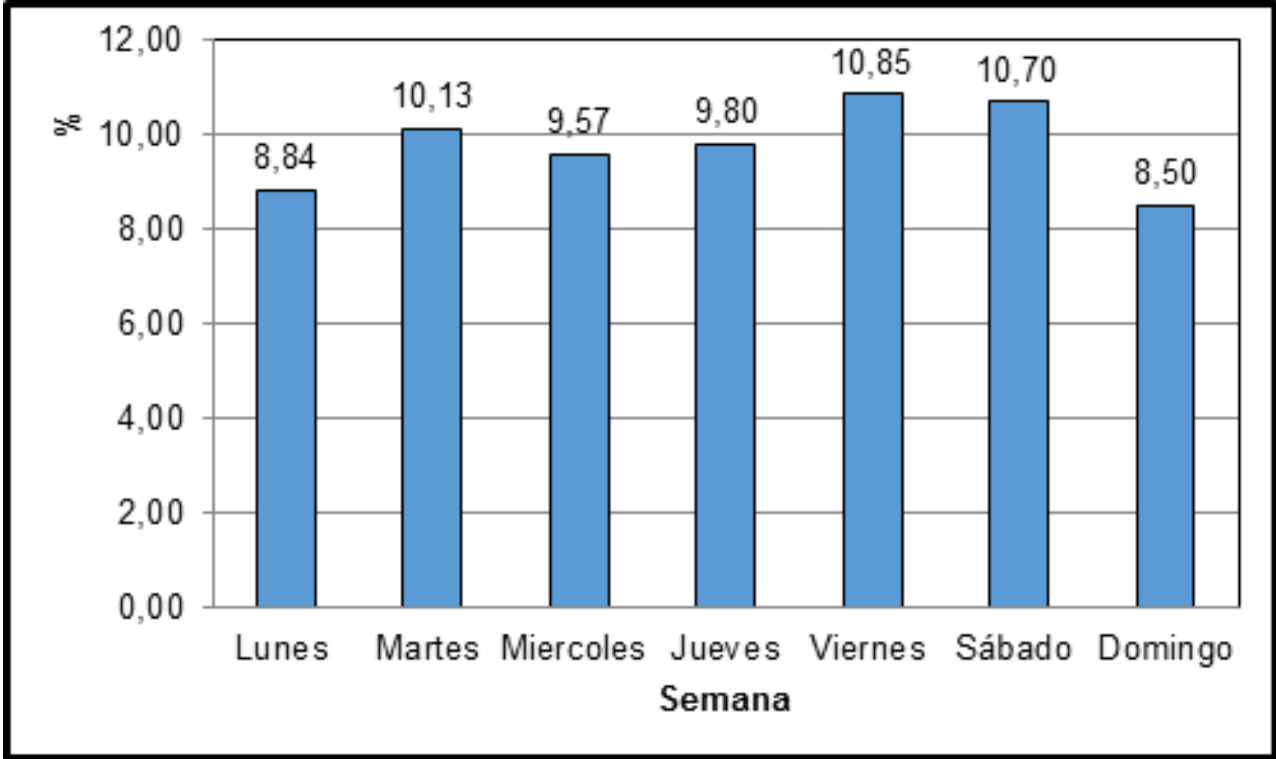

Figura 2. Porcentaje de residuos de plásticos en relación al per cápita.

Actitud ecológica de reciclar residuos de plásticos

Referente a la evaluación de la actitud ecológica de reciclar los residuos de plásticos que se expone en la Tabla 3, se destaca que no reciclan $61 \%$ de las casas representativas encuestadas; por lo que se analiza que gran parte de la población desconoce o no toma conciencia sobre las consecuencias que ocasiona estos materiales expuestos al aire libre o vertidos en fuentes de agua; puesto que sus componentes son derivados del petróleo y demora muchos años en degradarse.

Tabla 3. Actitud ecológica de reciclar los residuos de plásticos (\%)

\begin{tabular}{lcccc}
\hline & Lo arrojan en la calle & Lo reciclan & No reciclan & No opinan \\
\hline Reciclar & 25 & 5 & 61 & 9 \\
\hline
\end{tabular}

\section{Discusión}

\section{Caracterización de los residuos domiciliarios}

En cuanto a la caracterización de los residuos domiciliarios que se detalla en la tabla 1 , se determinó que en noviembre hubo incremento en la generación de residuos con $1591.77 \mathrm{~kg} / 50$ casas/ mes. También se determinó que en las características sobresalió los residuos orgánicos con $57.32 \%$ y se mantuvo los residuos inorgánicos con $39.21 \%$. Esto se debe a que durante la pandemia la población, se abasteció de productos básicos de primera necesidad y al finalizar el año se resalta la frecuencia a centros comerciales, restaurantes y otros lugares de comercio, lo que dio como resultado el incremento de los residuos de preferencia envases, 
bolsas plásticas y empaques. Este análisis se sostiene con Requena et al., (2021), quienes concluyen que durante la cuarentena de Covid-19, los residuos orgánicos pueden haber aumentado por una mayor frecuencia y cantidad de alimentos preparados en casa.

\section{Per cápita de residuos sólidos domiciliarios}

Respecto a la evaluación per cápita de los residuos domiciliarios que se detalla en la Figura 1, se determinó que el día jueves con $0.49 \mathrm{~kg} /$ habitante destacó con relación a los demás días. Esto se debe a que paulatinamente se impulsa la reactivación económica del país, lo que se muestra por la concurrencia a los mercados, centros de abastos $\mathrm{y}$ otros comercios resultando el incremento de residuos domiciliarios como materiales de plásticos, residuos de comida y otros con relación años anteriores, según la Municipalidad Provincial de Barranca (2018) menciona que los residuos sólidos domiciliarios presentan un volumen total mensual de $45.5 \mathrm{~m} 3$ equivalentes a $540 \mathrm{Tn}$./Mes; con una generación de residuos per cápita promedio diario equivale a $0.351 \mathrm{Kg}$.

\section{Porcentaje de residuos de plásticos por mes}

Concerniente al porcentaje de residuos de plásticos por mes que se indican en la Tabla 2, se resalta que en noviembre incrementó a $7.48 \%$ del total de $1560.77 \mathrm{~kg} / 50$ casas/ mes. Esto se debe que al finalizar el año se percibe que la población recurre a mercados, centros de abastos u otros lugares de comercio, lo cual influye en el incremento de estos residuos. Por lo que, es necesario tener en cuenta una adecuada disposición final de estos residuos de materiales de plásticos y conciencia ambiental; puesto que toman muchos años en degradarse y pueden ocasionar impacto negativo al ambiente y salud pública. Este análisis se sostiene con Canchari, y Iannacone (2021) quienes concluyen que, los residuos biocontaminados generados son mínimos, y tanto plásticos de un solo uso y los papeles desechables fueron mayores en comparación con la parte reciclable. Asimismo, Escobar et al., (2019) concluyen existe una alta probabilidad de exposición a los microplásticos transferidos por niveles tróficos, sobre todo para poblaciones que habitan cerca de las vías marítimas y próximas a áreas urbanas o industriales.

\section{Porcentaje de residuos de plásticos en relación al per cápita}

Obtenidos los resultados del porcentaje de residuos de plásticos en relación al per cápita que se muestra en la Figura 2, se aprecia que el día jueves sobresalió con $10.85 \%$ con relación a lo demás días. Por lo tanto, se establece como un indicador porcentual que se debe de tener en cuenta, tener conciencia ambiental y aplicar medidas adecuadas en la disposición final con el fin de que haya un tratamiento adecuado de estos residuos; puesto que su exposición al aire libre o en fuentes de agua ocasionan daños ambientales. Lo analizado se fundamenta con Chuiza-Rojas et al., (2020) quienes exponen que los plásticos convencionales obtenidos a partir del petróleo tardan de 100 a 1000 años en degradarse, causando grandes impactos al ambiente. 
Actitud ecológica de reciclar residuos de plásticos

Efectuado el análisis estadístico de la evaluación de la actitud ecológica de reciclar los residuos de plásticos que se observa en la Tabla 3 , se aprecia que destaca el $61 \%$ que no reciclan estos residuos. Por lo tanto, gran parte de la población desconoce o no tiene conciencia ecológica en la disposición final de los desechos de bolsas, envases u otros materiales plásticos; puesto que lo vierten al aire libre, ríos, playas etc., ocasionando daños ambientales al ecosistema que afectan directamente a la salud pública. Por este motivo, es necesario colocar estos residuos en tachos, no arrojarlos en las calles y usar las bolsas en lo necesario; es decir, darles un tratamiento de acuerdo con los planteamientos de la educación ambiental. De acuerdo a ello, sostiene Tito (2019) quien concluye que, los resultados indican que la educación ambiental contribuye significativamente a la intensión de reducir el uso de bolsas plásticas en la Institución Educativa Coronel Pedro Portillo Silva-Huaura.

\section{CONCLUSIONES}

Se determinó que en noviembre sobresalió con $7.48 \%$ de residuos de plásticos por mes; lo que quiere decir que de $100 \mathrm{~kg}$ de residuos domicilios $7.48 \mathrm{~kg}$ son de materiales de plásticos. Por lo tanto, este resultado se establece como un indicador porcentual, para que se tome conciencia ecológica al momento de reciclar estos residuos con el fin de reducir la contaminación ambiental y propagación de enfermedades.

También, se determinó que el $61 \%$ de la población no tiene actitud ecológica de reciclar los residuos de plásticos. Por lo que, desconocen las consecuencias que puede ocasionar si estos materiales no tienen un adecuado tratamiento; puesto que están hechos por componentes derivados del petróleo y que demora muchos años en degradarse. Por este motivo se debe reciclar en recipiente o bolsas de almacenamiento del mismo material.

\section{REFERENCIAS}

Canchari, F y Iannacone, J. (2021). Residuos Sólidos Municipales en el Centro Poblado de Madeán, distrito de Madeán, provincia de Yauyos, región Lima, Perú en época de Pandemia del Covid-19. Revista Científica PAIDEIA XXI, 11, 275-289. https://doi.org/10.31381/paideia.v11i2.4038

Cantanhede A., Monge G., Sandoval L. y Caycho C. (2005). Procedimientos Estadísticos para los estudios de Caracterización de Residuos Sólidos, Revista AIDIS de Ingeniería y Ciencias Ambientales: Investigación, Desarrollo y Práctica: Volumen 1, Número 1. Visto 25 de octubre 2021 http://www.revistas.unam.mx/ index.php/aidis/article/view/13553

Chuiza-Rojas, M., Rodríguez-Basantes, A y BritoMoína, H. (2020). Producción de láminas de plástico biodegradables a partir del almidón de arracaciaxanthorrhiza. Dominio de Las Ciencias, 6, 981-994. https://dominiodelasciencias.com/ ojs/index.php/es/article/view/1261

Escobar Condor, E. W., Izquierdo Villasante, Y., Macedo Riva, A., Remuzgo Panduro, G., y Huiman Cruz, A. (2019). Impacto de la ingesta de residuos plásticos en peces. Revista Kawsaypacha: Sociedad y Medio Ambiente, 4, 79-92. https://doi.org/10.18800/ kawsaypacha.201902.004

Flores, P. (2020). La problemática del consumo de plásticos durante la pandemia de la COVID-19. South Sustainability, 1(2), 1-9. https://doi. org/10.21142/SS-0102-2020-016

García L., y Rentería M. (2019). Plásticos en los océanos. Oficina de Información Ciencia y Tecnológica Para El Congreso de La Unión 
(INCYTU), 52(55). https://foroconsultivo. org.mx/INCyTU/documentos/Completa/ INCYTU_19-034.pdf

INEI.

CompendioEstadísticoLimaProvincias2016. CompendioEstadístico. INEI (Instituto Nacional de Estadística e Informática). Perú. Página 88. [Consulta: 01 de octubre del 2021]. https:// www.inei.gob.pe/media/MenuRecursivo/ publicaciones_digitales/Est/Lib1521/Libro.pdf

INEI. (2017). Censos Nacionales 2017: XII de Población, VII de Vivienda y III de Comunidades Indígenas. Documento Perú: Características de las viviendas particulares y los hogares. Acceso a servicios básicos. [Consulta: 01 de octubre del 2021]. INEI (Instituto Nacional de Estadística e Informática). Perú. Página https://www.inei. gob.pe/media/MenuRecursivo/publicaciones_ digitales/Est/Lib1538/Libro.pdf

Jaén, M., Esteve, P., y Banos-González, I. (2019). Los futuros maestros ante el problema de la contaminación de los mares por plásticos y el consumo. Revista Eureka Sobre Enseñanza y Divulgación de Las Ciencias, 16(1), 1-17. https://doi.org/10.25267/ RevEurekaensendivulgcienc.2019.v16.i1.1501

López-Aguirre, J., Pumaquero-Yuquilema, J., y Lopez-Salazar, J. (2020). Análisis de la contaminación ambiental por plásticos. Polo Del Conocimiento, 5(12), 725-742. https:// www.polodelconocimiento.com/ojs/index.php/ es/article/view/2139

Municipalidad Provincial de Barranca. (2018). Plan de desarrollo urbano de la ciudad de Barranca 2008-2018. pp. 1-14. Visto el 5 de octubre 2021 https://eudora.vivienda.gob.pe/observatorio/ PDU_MUNICIPALIDADES/BARRANCA/ PDU-BARRANCA.pdf
Pineda, E., De Alvarado E., y De Canales, F. (1994). Metodología de la investigación. Manual para el desarrollo de personal de salud. Metodología de La Investigación, 232. http://187.191.86.244/ rceis/registro/Metodologia $\% 20 \mathrm{de} \% 20 \mathrm{la} \% 20$ Investigacion $\% 20$ Manual $\% 20$ para $\% 20$ el $\% 20$ Desarrollo\%20de\%20Personal\%20de\%20Salud. pdf

Programa de las Naciones Unidas para el Medio Ambiente. (2019). Plásticos de un solo uso: Una hoja de ruta para la sostenibilidad. In Tecnology for Enviroment (Vol. 227, Issue 5). https:// cidoc.marn.gob.sv/documentos/plasticosde-un-solo-uso-una-hoja-de-ruta-para-lasostenibilidad/

Requena Sánchez, N., Carbonel Ramos, D., y Vallester, E. (2021). Generación y segregación de residuos sólidos domiciliarios durante la cuarentena por Covid-19 en Panamá, estudio de caso. Investigación y Pensamiento Crítico, 9(2), 16-24. https://doi.org/10.37387/ipc.v9i2.232

Tito Zúñiga, Y. (2019). Educación ambiental y la reducción del uso de bolsas plásticas en la institución educativa Coronel Pedro Portillo Silva de Huaura. Tesis para Optar el Título de Ingeniero Ambiental. Universidad Nacional José Faustino Sánchez Carrión. https://1library. co/title/educacion-ambiental-y-la-reducciondel-uso-de-bolsas-plasticas-en-la-institucioneducativa-coronel-pedro-portillo-silva-dehuaura 